Société d'histoire de la révolution de 1848 et des

révolutions du XIXe siècle

$37 \mid 2008$

L'ère victorienne revisitée

\title{
Des histoires contestées : l'histoire sociale de la Grande-Bretagne du XIXe siècle
}

Contested histories: nineteenth-century British social history

James Thompson

\section{OpenEdition}

Journals

Édition électronique

URL : http://journals.openedition.org/rh19/3506

DOI : $10.4000 /$ rh 19.3506

ISSN : $1777-5329$

Éditeur

La Société de 1848

Édition imprimée

Date de publication : 15 novembre 2008

Pagination : 15-35

ISSN : 1265-1354

Référence électronique

James Thompson, «Des histoires contestées : I'histoire sociale de la Grande-Bretagne du XIXe

siècle », Revue d'histoire du XIXe siècle [En ligne], 37 | 2008, mis en ligne le 01 décembre 2010, consulté le 19 avril 2019. URL : http://journals.openedition.org/rh19/3506 ; DOI : 10.4000/rh19.3506 


\section{JAMES THOMPSON \\ Des histoires contestées : l'histoire sociale de la Grande-Bretagne du XIXe siècle}

Cet article retrace les développements de l'histoire sociale de la GrandeBretagne du XIX ${ }^{\mathrm{e}}$ siècle au cours des trente dernières années. Il se divise en trois parties. Dans un premier temps, on traite des débats récurrents, vifs et parfois éprouvants sur la nature, l'avenir et l'existence de l'histoire sociale depuis la fin des années 1970. Ces accès d'introspection aiguë n'ont pas empêché une production considérable. On examinera ces écrits dans un second temps. Les descriptions et les évaluations du tournant culturel dominent une bonne partie des commentaires sur l'évolution de l'histoire sociale britannique : on y prêtera, dans un troisième temps, l'attention qui leur est due. Cependant, cet essai vise aussi à présenter une tableau plus ample des mutations de l'histoire sociale britannique au cours de la période, en la replaçant dans le contexte de la discipline dans son ensemble, et en s'intéressant à ses relations complexes avec l'histoire politique et avec l'histoire économique. Cet article s'achève par un bref coda sur l'héritage des trente dernières années, et présente certains des défis à venir.

Les panoramas de l'histoire sociale soulignent souvent un contraste entre un âge d'or d'efforts hérö̈ques au cours des années 1960 et 1970, et les évaluations plus hésitantes de sa vitalité au cours de la période qui a suivi. En terme de publications, on peut dire que ce récit surestime tout à la fois la domination de l'histoire sociale dans la période antérieure, et son déclin récent, quoique une bonne partie du débat porte sur où on place la frontière entre l'histoire sociale et l'histoire culturelle au cours des années 1980 et 1990. Il est cependant difficile de nier un changement de ton entre le projet collectif conquérant pour l'histoire sociale paru dans la célèbre édition spéciale du Times Literary Supplement en 1966 sur les «Nouvelles perspectives en histoire», et les débats du milieu des années 1990 sur la mort de l'histoire sociale, dans la revue Social History, entre autres. Cependant, cet article est 
consacré aux trente dernières années. Et, vers la fin des années 1970, le débat sur les accomplissements, l'identité et les possibilités de l'histoire sociale avait déjà eu lieu.

Les années 1960 et le début des années 1970 ont connu des développements significatifs dans l'écriture de l'histoire sociale britannique du XIX siècle. L'expansion de l'histoire sociale avait un certain nombre de sources. L'une d'entre elles était sans conteste d'origine marxiste. Les deux figures les plus éminentes de l'épanouissement de l'histoire sociale marxiste d'après-guerre furent Edward P. Thompson et Eric Hobsbawm. L'étude d'E.P. Thompson sur La formation de la classe ouvrière anglaisel présentait un récit vivant, et très influent, de cette formation. Pour E.P. Thompson, la classe était une «relation historique» et un "processus actif». Alors que «l'expérience de classe était largement déterminée par [...] les rapports de production ", la conscience de classe était «la façon dont ces expériences sont maniées en termes culturels ${ }^{2}$. Dans les années 1960 et 1970, la conception qu'avait E.P. Thompson du rôle des acteurs et de celui de la culture dans la formation de la classe constitua un modèle pour une production historique significative. Ses articles sur la discipline au travail et sur l'économie morale de la foule anglaise ont proposé des récits, par la suite souvent cités, de l'impact de l'industrialisation et de la formation du capitalisme ainsi que des résistances qu'ils ont suscités ${ }^{3}$.

Si E.P. Thompson fut sans doute le plus éminent des historiens britanniques du social travaillant dans une tradition marxiste, l'influence d'Eric Hobsbawm fut également significative. Le travail d'Eric Hobsbawm en histoire sociale et ouvrière témoignait d'un intérêt plus grand pour la quantification et pour l'histoire économique que celui d'E.P. Thompson. Les travaux rassemblés dans son recueil Labouring Men paru en 1964 abordaient en particulier les débats sur le niveau de vie des ouvriers pendant la révolution industrielle, et sur l'impact du nouveau trade-unionisme des années $1880^{4}$. Un ensemble ultérieur de travaux sur les Worlds of Labour ont confirmé cette vision de la fin du XIX ${ }^{e}$ siècle comme une époque de re-formation de la classe ouvrière, à la suite des accommodements du milieu du siècle avec le capitalisme libéral expliqués, en partie, par le rôle de l'«aristocratie ouvrière" ${ }^{5}$.

1. Edward P. Thompson, The Making of the English Working Class, New York, Vintage Books, 1963, traduction française La formation de la classe ouvrière anglaise, Paris, Gallimard /Le Seuil, 1988.

2. Edward P. Thompson, La formation..., ouv. cité. p. 14.

3. Edward P. Thompson, "Time, Work-Discipline and Industrial Capitalism", Past and Present, 1967, $\mathrm{n}^{\circ}$ 38, p. 56-97, traduction française Temps, discipline du travail et capitalisme industriel, Paris, La Fabrique, 2004 (1967); Edward P. Thompson, "The Moral Economy of the English Crowd in the Eighteenth Century", Past and Present, ${ }^{\circ}$ 50, mai 1971, pp. 76-136, traduction française "L'économie morale de la foule dans l'Angleterre du XVIII e siècle», dans Florence Gauthier, Guy-Robert Ikni [dir.], La guerre du blé au XVIII siècle, Montreuil, Éditions de la Passion, 1988, p. 31-92; Edward P. Thompson, Customs in Common, Londres, Merlin, 1991.

4. Eric J. Hobsbawm, Labouring Men: Studies in the History of Labour, Londres, Weidenfeld and Nicholson, 1964.

5. Eric J. Hobsbawm, Worlds of labour: Further Studies in the History of Labour, Londres, Weidenfeld and Nicholson, 1984. 
Eric Hobsbawm fit partie du comité éditorial originel de Past and Present, revue en bonne partie marxiste, qui constitua, à partir des années 1950, un débouché important pour les publications en histoire sociale. Ce fut dans Past and Present que parurent certains des articles importants des historiens marxistes britanniques - en particulier, le travail d'E.P. Thompson sur l'économie morale de la foule - mais elle ne se cantonna pas à la publication de travaux d'histoire sociale ouvertement marxistes. Leur prestige intellectuel et leurs ressources organisationnelles comptèrent beaucoup dans l'influence qu'eurent certains historiens marxistes sur la pratique de l'histoire sociale en Grande-Bretagne. Cependant, comme l'a noté Miles Taylor, le marxisme n'a jamais constitué la seule source du développement de l'histoire sociale en Grande-Bretagne. L'histoire sociale est issue de courants en histoire politique et économique, tout en s'étant construite en conflit avec les orthodoxies existantes. Elle s'est développée depuis les hauteurs de l'establishment universitaire en même temps que dans des avant-postes plus rebelles ${ }^{6}$.

Past and Present faisait partie d'un certain nombre de revues fortement liées à l'essor de l'histoire sociale dans les années 1960 et 1970. Social History et History Workshop (rebaptisé plus tard History Workshop Journal) naquirent au milieu des années 1970. "Revue d'historiens socialistes" dans sa forme d'origine, History Workshop était pour beaucoup issu du mouvement éponyme du "history workshop» ("l'atelier de l'histoire»), avec un engagement fort à écrire l'" histoire par en bas». Son comité éditorial de fondation avait des liens avec Past and Present, à travers l'historien de la société allemande sous le nazisme, Tim Mason, ancien directeur adjoint de Past and Present. Il comprenait d'autres figures de premier plan dans le développement de l'histoire sociale à l'université d'Oxford, comme Gareth Stedman Jones et Raphael Samuel. Il est clair que les réseaux de la nouvelle gauche ( $N e w$ Left») jouèrent un rôle dans la constitution de cette revue. Comme les ateliers annuels tenus à Ruskin College à Oxford, cette revue s'engageait à placer les «frontières de l'histoire plus près de la vie des gens». Elle voulait préserver «l'enracinement fort dans l'expérience de la classe ouvrière» des history workshops, mais aspirait à le faire sous une forme plus ouvertement théorique et internationaliste. Elle avait l'intention de faire progresser le «savoir démocratique» et de toucher un public large au-delà des seuls historiens de métier ${ }^{7}$.

Les engagements politiques et le désir de s'adresser à un public plus large étaient caractéristiques de l'esprit conquérant de l'histoire sociale au cours des années 1960 et 1970. Comme Miles Taylor nous le rappelle, cependant, cela n'était qu'une partie du tableau ${ }^{8}$. L'intérêt pour la vie quotidienne, qui

6. Miles Taylor, "The beginnings of modern British social history?", History Workshop Journal, $\mathrm{n}^{\circ} 43,1997$, p. $155-76$.

7. Editorial Collective, "History Workshop Journal", History Workshop, nº 1, 1976, p. 1 et 3.

8. Miles Taylor, "The beginnings of modern British social history?", idem, p. 155-156. 
est à la base d'une bonne part de l'histoire sociale, n'était pas le monopole de la seule gauche. L'objet institutionnel de certains travaux, notamment en histoire de la protection sociale (welfare history), exprimait une dette à l'égard des modes établies de l'histoire ouvrière et politique qu'il faut distinguer des travaux plus engagés qui ont fait l'objet d'un intérêt historiographique plus important. Néanmoins, ce qui semble clair est qu'une grande partie de cette période était caractérisée par une confiance partagée quant aux perspectives de l'histoire sociale. Cependant, à la fin des années 1970, certains signes suggérèrent que cet optimisme commençait à flancher.

En 1979, Tony Judt annonçait que "c'est une mauvais époque pour être un historien du social» ${ }^{9}$. L'article maintenant bien connu de Tony Judt se présentait volontairement et péremptoirement dans des termes d'opposition, mais il reflétait un processus plus large de réflexion. Un certain nombre d'historiens anglophones de premier plan, liés les uns aux autres, publièrent des critiques vives de cette sous-discipline à la fin des années 1970 et au début des années 1980. Leurs critiques n'étaient pas identiques, mais on pouvait y identifier des recoupements importants. En dépit de leurs subtiles différences, Geoff Eley et Keith Nield, Tony Judt et Gareth Stedman Jones voyaient tous un déficit théorique au cœur de l'histoire sociale ${ }^{10}$. C'était, en partie, une attaque contre des formes particulières de sociologie qui, selon ces critiques, occupaient une place centrale dans la façon dont l'histoire sociale était pratiquée. Ils tenaient notamment pour circulaires et problématiques les théories de la «modernisation» et les explications fonctionnalistes. En particulier, Tony Judt affirmait que l'histoire sociale dominante ne prêtait pas suffisamment attention aux événements et tombait dans un déterminisme qui occultait le rôle des acteurs. Là où ces auteurs se rejoignaient, cependant, c'était dans l'idée qu'une bonne partie de l'histoire sociale conventionnelle négligeait la politique et l'idéologie, et était mal armée pour s'acquitter de sa tâche essentielle : comprendre l'exercice du pouvoir.

Cette remise en question caractéristique de la période du tournant des années 1980 n'était pas, bien sûr, confinée à l'histoire sociale britannique, encore moins à celle du XIXe siècle. La liste établie par Tony Judt des revues souvent pécheresses comprenait les Annales, Comparative Studies in Society and History, le Journal of Interdisciplinary History et le Journal of Social History. Il épargnait en particulier E.P. Thompson (parmi d'autres) de ses critiques ${ }^{11}$. Geoff Eley et Keith Nield étaient préoccupés par les défauts de l'histoire sociale allemande comme ils l'étaient par les défauts des travaux sur la Grande-Bretagne. L'histoire sociale de la Grande-Bretagne du

9. Tony Judt, "A clown in regal purple: social history and the historians", History Workshop, $\mathrm{n}^{\circ} 7$, 1979, p. 66.

10. Geoff Eley and Keith Nield, "Why does social history ignore politics?", Social History, n ${ }^{\circ}$, 1980, p. 249-271; Gareth Stedman Jones, "From historical sociology to theoretical history", British Journal of Sociology, $\mathrm{n}^{\circ} 27,1976$, p. 295-305.

11. Tony Judt, "A clown in regal purple", art. cité, p. 89. 
$\mathrm{XIX}^{\mathrm{e}}$ siècle était pourtant largement au centre de ce processus général de reconsidération. Bien sûr, il y avait eu des précurseurs. Même à l'époque où l'histoire sociale était censée être au zénith, Eric Hobsbawm observait que "ses meilleurs praticiens [s'étaient] toujours sentis mal à l'aise avec cette discipline» ${ }^{12}$. Néanmoins, la fin des années 1970 et le début des années 1980 ont constitué un moment significatif dans le débat méthodologique sur la nature et les références de l'histoire sociale.

La relecture de cette littérature à trente ans d'écart révèle à la fois des changements et des continuités. La très longue polémique de Tony Judt contre la quantification inscrit clairement son article dans sa propre époque. On peut trouver des passages similaires dans la présentation contemporaine et très discutée de Lawrence Stone sur le «renouveau du récit» (revival of narrative ${ }^{13}$. L'article de Lawrence Stone exprimait, notamment, ce sentiment de déception qui suit les grandes espérances; c'était l'exemple vigoureux d'un désenchantement plus large quant au projet visant à dénombrer la réalité. Les décennies qui ont suivi ont connu une divergence croissante entre les méthodes et les intérêts des historiens du quantitatif et ceux du qualitatif. Les relations entre ces parties divorcées semblent aujourd'hui n'être pas tant marquées par l'hostilité que par l'indifférence : de moins en moins d'historiens anglophones appartiennent aux deux tribus.

À d'autres égards, cependant, les débats de la fin des années 1970 anticipaient des préoccupations plus tardives. Tony Judt diagnostiquait déjà l'influence funeste de Michel Foucault, qui détournait les historiens des intentions et du choix des acteurs dans l'analyse des idéologies du passé ${ }^{14}$. En prônant une confrontation plus profonde avec le pouvoir et l'inégalité, Geoff Eley et Keith Nield plaidaient pour une histoire fermement centrée sur l'explication et pleinement consciente de la politique de classe du capitalisme $e^{15}$. Plus tard, leurs réponses complexes au tournant culturel réitérèrent ce refus d'abandonner la causalité et la critique du capitalisme ${ }^{16}$. L'insistance sur l'économique et sur le politique dans ces analyses soulevait des questions pressantes sur l'identité et le caractère du social. Elles se poseraient de nouveau - quoique sous une forme différente - à la suite du tournant culturel, dans les querelles du milieu des années 1990 sur la fin de l'histoire sociale.

Nombre des réflexions les plus perspicaces, produites à la fin des années 1970 et au début des années 1980, sur la nature de l'histoire sociale provenaient d'historiens qui, comme Geoff Eley et Gareth Stedman Jones,

12. Eric. J. Hobsbawm, "From social history to the history of society", Daedalus, ${ }^{\circ}$ 100, 1971, p. $20-45$.

13. Lawrence Stone, "The revival of narrative: reflections on a new old history", Past and Present, $\mathrm{n}^{\circ} 85,1979$, p. 3-24.

14. Tony Judt, "A clown in regal purple", art. cité, p. 73.

15. Eley and Nield, "Why does social history ignore politics?", art. cité, passim.

16. Geoff Eley and Keith Nield, "Starting over: the present, the post-modern and the moment of social history”, Social History, n² 20, 1995, p. 355-64. 
se livraient à un dialogue critique avec la théorie marxiste. L'attaque célèbre livrée par Gareth Stedman Jones contre les modèles de contrôle social exprimait une opposition aux explications teintées de fonctionnalisme qui était clairement redevable à des critiques marxistes plus anciennes ${ }^{17}$. L'étude historiographique récente livrée par Geoff Eley sur les quarante dernières années révèle à quel point ses objections au projet de Bielefeld de Gesellschaftsgeschichte (histoire de la société) étaient enracinées dans une antipathie marxisante à l'égard des approches plus weberiennes ${ }^{18}$. La pensée même de Gareth Stedman Jones - comme nous allons le voir - s'éloignait de plus en plus des formulations marxistes reconnaissables alors que son insistance sur l'autonomie et sur l'impact des idées et du langage franchissait les frontières des conceptions même les plus culturalistes ou gramsciennes de la base et de la superstructure. Reste que les débats de la fin des années 1970 et des années 1980 concernant les questionnements marxistes, alors très vifs, étaient différents de ceux du milieu des années 1990.

Au milieu des années 1990 en effet, ceux qui proclamaient la fin de l'histoire sociale identifiaient les approches marxistes avec un projet moderniste d'histoire sociale rendu obsolète par l'avènement du post-modernisme. Patrick Joyce soutenait notamment, à la mode foucaldienne, qu'il fallait une histoire critique de la production du "social» plutôt que de nouvelles tentatives, vouées à l'échec, de chercher à tout absorber ${ }^{19}$. Convoquant un régiment éclectique de théoriciens, Patrick Joyce soutenait que l'histoire sociale incarnait une conception moderniste des catégories d'analyse structurelles et stables qui n'étaient pas à même de saisir le jeu de différence qui se manifeste dans la négociation et la constitution des identités. Sans surprise - vu son travail antérieur -, c'était sur le rôle de la classe que Patrick Joyce concentrait son attaque envers les procédures et les postulats de l'histoire sociale. D'après Patrick Joyce, malgré tout l'intérêt qu'Edward P. Thompson avait porté à la culture et à la politique, la tradition thompsonienne d'histoire sociale était fondée sur un modèle de formation de la classe dans lequel la conscience était modelée par l'expérience. Empruntant à la critique formulée par Joan Scott de "la preuve de l'expérience», Patrick Joyce disait avec insistance que l'histoire sociale établie s'appuyait sur une écriture du moi trop cohérente et univoque, et qu'elle était incapable de prendre en compte les régimes discursifs fragmentés et divers. Pour Patrick Joyce, la classe fonctionnait comme le concept fondamental de l'histoire sociale conventionnelle, reflétant son adhésion à un modèle explicatif fondé sur la «réalité» de l'économique. Selon

17. Gareth Stedman Jones, "Class expression versus social control? A critique of recent trends in the social history of 'leisure", History Workshop Journal, n 4, 1977, p. 162-70.

18. Geoff Eley, A Crooked Line: From Cultural History to the History of Society, Ann Arbor (Michigan), University of Michigan, 2005.

19. Patrick Joyce, "The end of social history?", Social History, n² 20, 1995, p. 73-92. 
lui, la logique du post-modernisme sapait les fondements de tout le projet d'histoire sociale.

Ce défi lancé par Patrick Joyce aux historiens du social dans les années 1990 comprenait des différences évidentes avec les manifestes diffusés autour de 1980. Alors que Tony Judt s'était alarmé de l'impact nouveau de Michel Foucault sur les historiens, Patrick Joyce déplorait leur incapacité à intégrer avec plus d'enthousiasme les implications des thèses de Michel Foucault sur le pouvoir et le savoir. Il y avait cependant des parallèles moins remarqués entre les deux. Patrick Joyce présentait la politique comme l'arène dans laquelle le sujet libéral était construit et était la source-clé des récits fondateurs du moi moderne ${ }^{20}$. En fait, le retour du politique était au cœur du programme de Patrick Joyce, comme il l'était chez ses précurseurs, quoique sous des traits différents. En effet, à la suite de Michel Foucault, son récit de la naissance du "social» mettait l'accent sur la gouvernementalité dans la mesure des populations et dans la définition d'une sphère du social, essentielle à l'émergence de l'idéal libéral du sujet autonome. Depuis son travail doctoral sur le conservatisme populaire dans le Lancashire, la politique, à la fois formelle et informelle, a été, pour Patrick Joyce, fondamentale pour comprendre la cohésion de l'Angleterre de la fin de l'époque victorienne ${ }^{21}$. On peut en partie voir ces arguments vivifiants sur la fin de l'histoire sociale comme une variation foucaldienne sur le thème plus ancien de l'incapacité de cette histoire sociale à englober la nature et l'impact du politique.

Cela ne fut pas, bien sûr, l'unique débat des années 1990. Comme William Sewell l'a justement noté, le passage de l'histoire sociale à l'histoire culturelle avait commencé dès le début des années 1980, sous l'infuence, entre autres, des analyses féministes du genre et des ramifications théoriques du tournant linguistique. Les historiens de la Grande-Bretagne étaient très impliqués dans les différents camps du conflit. William Sewell suggère que l'opposition au tournant culturel a été plus grande en Grande-Bretagne qu'aux ÉtatsUnis, en raison de la place plus importante occupée par l'histoire marxiste en Grande-Bretagne, et en raison de la centralité particulière de la classe pour l'historiographie et pour les historiens britanniques ${ }^{22}$. Cependant, les attaques contre les tournants culturel et linguistique dans les études britanniques venaient des États-Unis comme du Royaume-Uni. Tant William Sewell que Geoff Eley tendaient à sous-estimer la contribution non-marxiste à l'histoire sociale britannique. Repenser le rôle des classes sociales dans l'histoire britannique était souvent, comme le reconnaissent William Sewell et Geoff Eley, un projet de la gauche, façonné par le contexte politique sombre des années

20. Comme cela est développé dans Patrick Joyce, The Rule of Freedom: Liberalism and the Modern City, Londres, Verso, 2003.

21. Voir en premier lieu Patrick Joyce, Work, Society and Politics: the Culture of the Factory in Later Victorian England, Brighton, Harvester Press, 1980.

22. William H. Sewell, Logics of History: Social Theory and Social Transformation, Chicago, University of Chicago Press, 2005, p. 66-67. 
1980, et inspiré en partie par le désir de retrouver et de faire revivre des idées qui permettraient à un large centre-gauche de contester, armes théoriques en main, la pratique thatchérienne. La défense du tournant linguistique présentait une variété d'aspects et reflétait des préoccupations disparates : en fait, il faisait plus sens de parler de "tournants linguistiques» au pluriel plutôt que d'y voir une tendance théorique unique. L'approche linguistique proposée par Gareth Stedman Jones devait plus à l'intérêt pour la contextualisation et les intentions des acteurs propres à l'histoire intellectuelle de l'école de Cambridge qu'aux poussées déconstructives du post-structuralisme. Alors que pour Patrick Joyce, Michel Foucault semblait souvent faire partie de la solution, pour Gareth Stedman Jones il est devenu de plus en plus manifeste qu'il faisait partie du problème ${ }^{23}$. Sans surprise, des conceptions conflictuelles de ce qu'on appelait «les» Lumières sous-tendaient ces points de vue opposés. Pour Patrick Joyce, l'héritage des Lumières était l'auto-régulation des subjectivités modernes. Pour Gareth Stedman Jones, en revanche, c'étaient les Lumières qui avaient révélé l'image irrésistible d'un monde sans pauvreté, dans une combinaison séduisante de vertus libérales et républicaines ${ }^{24}$.

Au cours des dernières années, les débats théoriques ont perdu en vitalité et en férocité. La mode dominante dans les études britanniques est véhiculée par l'adoption de plus en plus fréquente de l'étiquette "histoire sociale et culturelle" par les revues, les sociétés savantes et les séminaires. La diffusion de cette description en dit long sur le développement des études historiques depuis l'époque où les départements et les séminaires naviguaient couramment sous les couleurs de l'" histoire sociale et économique». Nous réfléchirons plus loin au changement de relation entre l'histoire sociale et l'histoire économique. La popularité actuelle des appels à l'« histoire sociale et culturelle" peut être diversement interprétée. Elle témoigne d'une réticence partagée, malgré les polémiques des années 1990, à jeter le terme "social» avec l'eau du bain, tout en reconnaissant l'ascension irrésistible de l'histoire culturelle. La dernière décennie a certes vu un certain nombre d'historiens de premier plan - dont certains sont étroitement associés à la nouvelle histoire culturelle - insister sur la nécessité de garder une conception du social ${ }^{25}$. Geoff Eley y voit une caractéristique générationnelle, et affirme que les jeunes historiens sont plus heureux de combiner les apports de l'histoire sociale et de l'histoire culturelle que les chercheurs de sa génération, qui étaient formés comme historiens du social par les héritages de $1968{ }^{26}$. Il y a sans doute

23. Gareth Stedman Jones, "The determinist fix: some obstacles to the further development of the linguistic approach to history in the 1990", History Workshop Journal, n ${ }^{\circ} 42,1996$, p. 19-35.

24. Patrick Joyce, The Rule of Freedom, ouv. cité; Gareth Stedman Jones, An End to Poverty? A historical debate, Londres, Profile Books, 2004, traduction française La fin de la pauvreté? Un débat historique, Alfortville, Ėre, 2007.

25. Victoria E. Bonnell and Lynn Hunt (eds.), Beyond the Cultural Turn: New Directions in the Study of Society and Culture, Berkeley (California), University of California Press, 1999, p. 11.

26. Geoff Eley, A Crooked Line, ouv. cité, p. 283. 
un facteur de génération dans les tendances historiographiques, bien qu'il soit difficile de le spécifier avec précision. Il se peut que les jeunes historiens britanniques soient moins intéressés à débattre explicitement de la relation entre l'histoire culturelle et l'histoire sociale. Cela n'est peut-être pas, cependant, parce qu'ils ont transcendé ou résolu de quelque manière les questions méthodologiques qui se posent. Alors que l'essor de l'histoire culturelle a favorisé une sensibilité à certains types de théorie, elle n'a pas forcément élargi la formation théorique reçue par les historiens. Comme sous-discipline établie, l'histoire culturelle repose sur des présupposés qui sont souvent acquis tout-faits, et ne résultent plus de la difficile réflexion qu'exigeaient les odyssées intellectuelles entreprises par ses pionniers.

Certains commentateurs, notamment William Sewell, ont lié l'essor de l'histoire culturelle à certains développements du capitalisme contemporain : les historiens culturels seraient comme des apprentis rois mages dans la mystification des nouvelles inégalités voulues par la logique du capitalisme mondialisé ${ }^{27}$. Dans cette perspective, l'intérêt des historiens pour la circulation des représentations ne servirait qu'à détourner des réalités économiques contemporaines de la pauvreté et de l'exploitation. Cela constitue une forme trop réductrice de l'histoire intellectuelle, une forme qui doit historiciser plus pleinement sa compréhension du capitalisme contemporain. Cependant, cela nous rappelle que la Grande-Bretagne du XIX siècle était une économie en développement dans laquelle, comme Simon Szreter et d'autres l'ont montré récemment, l'industrialisation et l'urbanisation ont abaissé l'espérance de vie, dans certaines zones, à des niveaux inconnus depuis la Peste noire ${ }^{28}$. En fait, les liens plus anciens entre l'histoire sociale et l'histoire économique n'ont pas disparu au cours des dernières années, bien que les échanges entres les différents types d'histoire sociale n'aient pas toujours été aussi ouverts qu'on aurait pu l'espérer. En considérant, comme nous le ferons, l'influence du tournant culturel, la question de l'économique ne doit pas être négligée.

La question de l'avenir de l'histoire sociale était liée à des enjeux méthodologiques fondamentaux, en particulier ceux touchant les structures et les explications en histoire. Les tournants linguistique et culturel étaient rétifs à la réification des catégories par lesquelles les structures sociales étaient d'ordinaire délimitées. Les historiens britanniques préféraient de plus en plus s'attacher à la construction des identités sociales et à leur contestation plutôt que de cartographier la société selon des axes sociologiques prédéterminés. Il est important ici tout à la fois de reconnaître les apports et les idées légués par les approches discursives, et de faire la distinction entre ces phénomènes proches mais différents que sont les tournants culturel et linguistique. $\mathrm{Ni}$

27. William Sewell, Logics of History, ouv. cité, p. 62.

28. Simon R. Szreter and Graham Mooney, "Urbanization, mortality, and the standard of living debate: new estimates of the expectation of life at birth in nineteenth-century British cities", Economic History Review, $\mathrm{n}^{\circ}$ 51, 1998, p. 84-112. 
l'intérêt pour la micro-histoire, ni l'insistance sur la liberté individuelle dans la construction du sens ne sont des conséquences inévitables de l'adoption d'une démarche linguistique.

L'évaluation des effets des tendances récentes sur la notion de «structure sociale» dépendent ainsi de ce qu'on entend par structure. On a utilisé la métaphore de la structure pour avancer des arguments très différents. Elle peut simplement faire référence à des régularités observables, par exemple dans la répartition des revenus et des opportunités offertes par la vie. On peut l'utiliser pour nommer des acteurs collectifs réels ou potentiels. De façon plus générale, l'imagerie de la structure articule les conséquences à grande échelle de l'action individuelle à travers le temps, et les contraintes qui pèsent sur elle. Les tournants linguistique et culturel ont certainement eu de profondes conséquences pour la seconde de ces conceptions, compliquant les récits de l'émergence et de la persistance de l'action collective, et soulignant l'irréductibilité du politique. Cependant, reconnaître que nos catégories d'analyse sont des constructions ne doit pas empêcher de les appliquer au passé. Étant donné l'absence de toute position de Sirius au-dessus du monde du discours, nous ne pouvons que travailler avec ce que nous avons. Des régularités et des relations convaincantes dans l'histoire sociale et économique doivent être reconnues. Il faut analyser les dimensions globales et collectives des interactions humaines - ces circonstances que nous ne choisissons pas, en tout cas pas seuls. Les tentatives faites pour mener l'analyse sociale après les tournants culturel et linguistique, comme celle de William Sewell, ont eu tendance à modifier la métaphore, en faisant un large usage de la notion de Wittgenstein selon laquelle le mode de vie est incarné par des jeux de langage ${ }^{29}$. Si, en pratique, les historiens ont donc trouvé difficile de se passer du social en général, on peut dire autant pour une certaine acception de la «structure sociale».

De la même façon, de grandes questions se nichent dans les affrontements sur l'explication qui s'expriment dans les débats sur le statut de l'histoire sociale. Les versions les plus dures de l'histoire culturelle interprétative rejettent, comme tout simplement inaccessible, l'ambition explicative de l'histoire sociale. Plus largement, on n'accorde plus à l'économique et à l'expérimental le pouvoir d'explication qu'on leur attribuait auparavant. Les attitudes et les approches sur les questions de causalité varient de manière complexe au sein de l'histoire culturelle, et on ne peut distinguer de credo unique. Il faut se réjouir d'une subtilité plus grande, du rejet résolu du déterminisme et de la place accordée à la contingence. Cependant, il est moins sain qu'on se soit détourné des questions de causalité. En ce sens, il est temps de renouveler les débats antérieurs sur le caractère de l'explication historique.

Cette partie s'est intéressée aux conflits explicites et récurrents sur la viabilité et la signification de l'histoire sociale. Ces débats ont largement façonné

29. Par exemple, William H. Sewell, Logics of History, ouv cité, p. 337-338. 
les tendances historiographiques qui sont l'objet de notre deuxième partie. Plutôt que de tenter une énumération exhaustive des sous-champs toujours plus nombreux au sein de cette sous-discipline, nous chercherons à identifier les grands développements qui ont modelé l'histoire sociale britannique.

La transformation la plus spectaculaire dans l'écriture de l'histoire sociale britannique au cours des trente dernières années concerne le concept-clé de l'histoire sociale britannique des années 1960 et 1970 : la classe. On a appelé ces développements le "déclin de la classe", et ils sont étroitement liés aux controverses théoriques résumées ci-dessus ${ }^{30}$. Bien qu'il soit impossible de nier qu'on s'est éloigné ces dernières années des récits antérieurs de la formation des classes, la manière et le degré auquel cette question a été repensée ont varié entre les différents aspects de l'histoire sociale. De plus, les origines de cette distanciation sont complexes et loin d'être confinées à des questions méthodologiques.

Dans les années 1970, une bonne partie de l'histoire sociale du XIX ${ }^{\mathrm{e}}$ siècle se préoccupait de réconcilier la modernité économique et sociale de la Grande-Bretagne avec le caractère consensuel et obstinément libéral de sa vie politique. On consacra des efforts considérables à explorer les divisions parmi les ouvriers qualifiés, en particulier en termes de compétence et de religion, afin de comprendre ce qui était souvent vu comme l'émergence tardive d'un mouvement ouvrier politique indépendant ${ }^{31}$. Plus qu'il ne confirmait l'existence d'une aristocratie du travail séparée et se livrant à la collaboration de classe, ce travail eut clairement pour impact de démontrer l'importance durable d'un ensemble complexe de divisions parmi les travailleurs britanniques, comme la qualification, la religion, l'origine ethnique et l'appartenance régionale ou locale ${ }^{32}$. Les tentatives faites pour trouver des corrélations directes entre la position sociale et les tendances politiques semblaient irrémédiablement compromises. L'historiographie établie de la re-formation de la classe ouvrière après 1870 était de plus en plus critiquée.

L'histoire des femmes fut sans aucun doute un élément majeur pour repenser l'histoire sociale dans les années 1970. Les récits héroïques d'E.P. Thompson et d'Eric Hobsbawm avaient très peu de choses à dire sur les expériences

30. Pour un texte plus complet, voir James Thompson, "After the fall: class and political language in Britain 1780-1900", Historical Journal, $\mathrm{n}^{\circ}$ 39, 1996, p. 785-806.

31. Voir, par exemple, Geoffrey Crossick, An Artisan Elite in Victorian Society: Kentish London, 1840-80, Londres, Croom Helm, 1978; Robert Q. Gray, The Labour Aristocracy in Victorian Edinburgh, Oxford, Clarendon Press, 1976; Robert Q. Gray, The Labour Aristocracy in Nineteenth-Century Britain, c. 1850-1900, Londres, Macmillan, 1981.

32. Ces développements sont bien présentés dans Alastair. J. Reid, Social Classes and Social Relations in Britain, 1850-1914, Basingstoke, Macmillan, 1992. 
et les identités des femmes, soulignait-on avec force. Le fait qu'on s'intéresse d'abord aux relations sur le lieu de travail posait problème pour mettre au jour la vie des femmes, à une époque de plus en plus caractérisée par la séparation entre les sphères publique (masculine) et privée (féminine). Il faut cependant rappeler que l'impact de l'histoire des femmes, et du genre comme catégorie d'analyse, était loin d'être simple. Dans leur célèbre étude Family fortunes, Leonore Davidoff et Catherine Hall liaient fortement l'idéologie de la domesticité à la formation d'une middle class plus sûre d'elle, déterminée à se différencier des mondes familiaux moins respectables des patriciens et des plébéiens; l'attention portée aux relations entre hommes et femmes n'était pas incompatible avec un accent mis sur l'importance des classes sociales ${ }^{33}$. Cela dit, l'intérêt croissant pour l'histoire des femmes puis pour l'histoire du genre devait révéler les limites de bien des écrits sur la classe. Pareillement, l'émergence d'un ensemble de travaux démontrant l'importance des divisions de genre contribuait à miner le caractère central traditionnellement accordé aux classes sociales dans l'histoire sociale britannique. De façon plus fondamentale, les impératifs théoriques manifestes dans les plaidoyers pour l'usage du genre comme catégorie d'analyse s'accommodaient mal des démarches en vigueur quant aux classes sociales en histoire sociale britannique ${ }^{34}$. La critique la plus influente, par exemple, du recours à l'«expérience" propre à une grande partie de l'histoire sociale émanait de la principale partisane de l'analyse de genre ${ }^{35}$.

Ce retour réflexif sur les classes sociales dans la Grande-Bretagne du XIX siècle n'était pas sans liens avec les développements de l'histoire économique, en particulier dans ses phases antérieures. L'idée ancienne du "décollage», c'est-à-dire du big-bang de l'industrialisation, popularisée par le travail de Walt Rostow sur les étapes de la croissance économique, était soumise à un feu nourri de critiques techniquement impressionnantes ${ }^{36}$. L'effet d'ensemble de cette littérature était de décentrer la révolution industrielle dans l'histoire sociale britannique. En révisant à la hausse les estimations de la croissance urbaine et de l'urbanisation au début du XVIII e siècle, et en minimisant tant l'ampleur que l'importance de l'industrialisation plus tardive, les historiens de l'économie mettaient en cause les prémisses interprétatifs de nombreux historiens de la formation des classes au début du XIX siècle. Au sein de l'histoire économique, les points de vue révisionnistes de Nicholas Crafts ne restèrent pas sans réponse; et certains travaux récents de démographie histo-

33. Leonore Davidoff et Catherine Hall, Family Fortunes: Men and Women of the English Middle Class 1780-1850, Londres, Hutchinson, 1987. Voir, sur cet ouvrage et sa portée, l'article de Christina de Bellaigue dans ce numéro.

34. Joan W. Scott, Gender and the Politics of History, New York, Columbia University Press, 1988.

35. Joan W. Scott, “The evidence of experience”, Critical Inquiry, n 17, 1991, p. 773-797.

36. Walt Whitman Rostow, The Stages of Economic Growth: a Non-Communist Manifesto, Cambridge, Cambridge University Press, 1960, traduction française Les étapes de la croissance économique, Paris, Le Seuil, 1963. Louvrage classique du révisionnisme achevé est celui de Nicholas Crafts, British Economic Growth during the Industrial Revolution, Oxford, Clarendon Press, 1985. 
rique ont insisté sur l'impact massif de la croissance industrielle et urbaine sur l'espérance de vie ${ }^{37}$. Néanmoins, les explorations quantitatives de l'histoire économique ont joué leur rôle pour diminuer la centralité de la notion de classe dans l'histoire sociale britannique du XIXe siècle.

Rétrospectivement, cependant, c'est peut-être le travail sur la politique et le langage politique qui eut le plus grand impact. En particulier, l'essai de Gareth Stedman Jones, "Rethinking Chartism”, a eu une grande influence ${ }^{38}$. Gareth Stedman Jones soutenait que les interprétations sociales dominantes du chartisme avaient échoué à expliquer la nature politique de son programme. Sans nier le caractère majoritairement populaire de leur base, Gareth Stedman Jones soutenait que les chartistes comprenaient leur situation difficile en des termes essentiellement politiques. Enracinant leur critique dans les termes hérités du radicalisme du dix-huitième siècle, les chartistes décrivaient l'État comme une cabale aristocratique réduisant à la mendicité le peuple par les impôts, d'où la nécessité d'une réforme politique qui rende l'exécutif plus à même de répondre aux besoins de la population. En mettant l'accent sur la primauté du politique, Gareth Stedman Jones faisait des reproches importants aux historiens du social qui cherchaient à expliquer la politique par les changements sociaux. De façon plus controversée, cependant, son essai amorçait aussi un tournant "populiste» (populist) dans les études du XIX ${ }^{\mathrm{e}}$ siècle où l'approche linguistique, sous différentes formes, était utilisée pour affirmer que c'était la catégorie du peuple plutôt que celle de la classe qui représentait l'identité collective essentielle dans la société du $\mathrm{XIX}^{\mathrm{e}}$ siècle. Certains des travaux qui ont suivi, en particulier ceux de Patrick Joyce, étaient assez critiques de Gareth Stedman Jones, et s'écartaient de sa méthodologie, caractéristique de Cambridge; mais l'héritage de Languages of class demeure indubitable. Son effet sur l'étude de la politique populaire, tout comme son impact sur les travaux sur les classes sociales, ont été profonds.

Si Gareth Stedman Jones a aidé à susciter un intérêt pour la catégorie du «peuple», c'est Patrick Joyce qui a le plus fait pour faire du "populisme» (populism) un récit alternatif téméraire du XIX ${ }^{\mathrm{e}}$ siècle britannique. Dans ses études importantes, Visions of the People et Democratic Subjects, Patrick Joyce a dressé un tableau de l' "Angleterre industrielle» où la catégorie de "peuple» était la forme d'appartenance la plus pénétrante et la plus convaincante pour les ouvriers des années 1840 à la Première Guerre mondiale ${ }^{39}$. S'appuyant sur la compréhension communautaire des relations sociales proposée dans

37. Maxine Berg et Pat Hudson, "Rehabilitating the industrial revolution", Economic History Review, 45, 1992, p. 24-50.

38. Cet essai est paru en plusieurs versions. La plus complète est Gareth Stedman Jones, "Rethinking Chartism" dans son Languages of Class: Studies in English Working Class History, 1832-1982, Cambridge, Cambridge University Press, 1983. Traduction française partielle par Daniel Argelès et Philippe Minard, "Repenser le chartisme", Revue d'histoire moderne et contemporaine, n 54-1, 2007-1, p. 7-68.

39. Patrick Joyce, Visions of the People: Industrial England and the Question of Class, 1848-1914 Cambridge, Cambridge University Press, 1991 ; Patrick Joyce, Democratic Subjects: the Self and the Social in Nineteenth Century England, Cambridge, Cambridge University Press, 1994. 
Work, society and politics, Patrick Joyce a analysé un grand ensemble de pratiques discursives - de l'histoire des dialectes à l'architecture des usines -, soutenant que, alors que les classes étaient loin d'être complètement absentes, c'est l'image d'un peuple industrieux, souvent opposée à celle d'une élite oisive, qui était la plus juste pour évoquer les gens des usines de l'Angleterre du XIX siècle.

À la lumière des publications ultérieures de Patrick Joyce, Visions of the people apparaît maintenant comme un travail de transition, déchiré entre la volonté de présenter le peuple comme une alternative à l'analyse traditionnelle en termes de classes sociales, et proclamant l'obsolescence de tels élans totalisants. Adoptant ouvertement une démarche plus systématiquement post-structuraliste, Democratic subjects, qui a suivi, racontait la formation de deux hommes - l'auteur dialectal de Lancaster Edwin Waugh et l'homme politique libéral du Nord John Bright - afin d'éclairer la relation entre le moi et le social dans l'Angleterre du XIX ${ }^{e}$ siècle. S'inspirant en partie au travail de Jacques Rancière, Patrick Joyce soutenait que l'identité de Waugh provenait d'une "religion de l'humanité" qui était inclusive plutôt qu'exclusive, et qui mettait l'accent sur l'harmonie plutôt que sur la discorde sociale ${ }^{40}$. Joyce insistait sur les points communs entre l'autodidactisme de Bright et de Waugh, en notant leur tendance partagée à insister sur l'importance du franc-parler indépendant. Le récit (le narrative), bien qu'étant déjà un terme important dans Visions of the people, était maintenant présenté comme le moyen principal par lequel réussir la négociation entre le moi et la cartographie du social. Son mode principal dans l'Angleterre du XIX siècle - en particulier tel qu'il était révélé dans les biographies de Bright - était celui du mélodrame. Comme le suggère Rosalind Crone, l'accent mis sur le mélodrame devait émerger comme un leitmotiv important dans l'histoire culturelle victorienne ${ }^{41}$.

Pour préciser l'importance du concept de classe dans l'histoire sociale de la Grande-Bretagne du XIX ${ }^{e}$ siècle, un indicateur est fourni par la publication en 1990 de la Cambridge Social History of Britain en trois volumes, sous la direction de F. M. L. Thompson ${ }^{42}$. Comme je l'ai noté ailleurs, les 1400 pages de cet ouvrage en trois volumes ne contenaient pas de chapitre explicitement consacré à la classe ${ }^{43}$. En cela, et dans son architecture d'ensemble, la Cambridge Social History en disait long sur les tendances en histoire sociale britannique. Le volume consacré aux "régions et aux communautés» intégrait utilement le vaste ensemble des études locales résultant de l'essor de

40. Patrick Joyce, Democratic subjects, ouv. cité, p. 28-29. Patrick Joyce s'inspire de Jacques Rancière, La nuit des prolétaires : archives du rêve ouvrier, Paris, Hachette, 1981.

41. Voir l'article de Rosalind Crone sur l'histoire culturelle.

42. F.M.L. Thompson (ed.), The Cambridge Social History of Britain, 1750-1950, Cambridge, Cambridge University Press, 1990, 3 volumes.

43. James Thompson, «L'histoire sociale de la Grande-Bretagne du XIX ${ }^{e}$ siècle entre crise et renouveau", Histoire et société. Revue européenne d'histoire sociale, n² 2, 2002, p. 20-36. 
l'histoire sociale des précédentes décennies. Le volume consacré aux «agencements sociaux» et aux «institutions» reconnaissait à juste titre l'émergence de sujets comme le crime et l'éducation comme des piliers de l'histoire sociale. Il faut noter que les classes n'avaient pas complètement disparu : mais un certain nombre de recensions exprimaient le sentiment qu'elles étaient certainement devenues marginales ${ }^{44}$. Dans sa longue étude sur «les villes et les campagnes", le responsable de l'ouvrage prétendait que ce n'était que pendant l'entre-deux-guerres que la "société urbaine s'était éloignée... d'une société alvéolaire pour devenir une société de classes». La Cambridge Social History était marquée par les débats plus théoriques des années 1980 - elle incluait un chapitre sur le travail par Patrick Joyce - mais ce n'était pas principalement ce qui déterminait son traitement des classes sociales.

Les ouvrages collectifs en plusieurs volumes relèvent rarement d'une seule perspective méthodologique, et la Cambridge Social History ne faisait pas exception. Il est cependant intéressant de considérer les vues de son principal artisan, F.M.L. Thompson. Alors que c'était déjà, en 1990, un historien distingué avec de nombreuses publications, F.M.L. Thompson avait pendant quelque temps défendu une vision de la société britannique au XIX ${ }^{\mathrm{e}}$ siècle bien exprimée par le titre de son ouvrage, The Rise of Respectable Society ${ }^{45}$. Pour F.M.L. Thompson, la prédominance croissante de l'idéal de respectabilité fournissait la meilleure base pour comprendre ce qui soudait la société du XIX ${ }^{e}$ siècle. Critique mordant des modèles de contrôle social, F.M.L. Thompson ne voyait pas l'adhésion à la respectabilité comme une forme de conscience erronée de la part des travailleurs; il insistait plutôt sur les avantages et les attraits réels présentés par la respectabilitét ${ }^{46}$. À certains égards, en organisant l'histoire sociale du XIX ${ }^{\mathrm{e}}$ siècle autour du triomphe d'un idéal, F.M.L. Thompson rappelait le travail de Harold Perkin. Dans son célèbre livre, The Origins of Modern English Society, celui-ci avait soutenu que le XIX ${ }^{e}$ siècle avait connu deux changements éthiques majeurs : le passage d'une éthique aristocratique à une éthique entrepreneuriale dans les années 1830 , et celui d'une éthique entrepreneuriale à une éthique professionnelle dans les années $1880^{47}$. Bien qu'elle ne fût certainement pas présentée comme telle, l'approche de Harold Perkin pouvait grosso modo être classée comme wéberienne. En fait, on pourrait suggérer que dans le travail de F.M.L.Thompson également, l'approche wéberienne fournissait implicitement une source de la distance prise vis-à-vis des modes d'écriture classistes.

Ces trente dernières années ont assurément produit des changements

44. Voir, par exemple, Geoffrey Crossick, "Consensus, order and the social history of modern Britain", Historical Journal, $\mathrm{n}^{\circ} 35,1992$, p. 945-951.

45. F.M.L. Thompson, The Rise of Respectable Society: a Social History of Victorian Britain, 18301900, Londres, Fontana, 1988.

46. F.M.L. Thompson, "Social control in Victorian Britain", Economic History Review, n 34, 1981, p. 193-208.

47. Harold Perkin, The Origins of Modern English Society, 1780-1880, Londres, Routledge, 1969. 
remarquables dans la conception des classes au sein l'historiographie de la société britannique au XIX ${ }^{\mathrm{e}}$ siècle. Elles ne les ont cependant pas enterrées : la classe sociale est considérée comme l'une des diverses identités produites par le discours. Comme le langage de "l'identité" a remplacé celui de la "conscience», c'est de plus en plus l'identité des classes moyennes qui a été étudiée de plus près. Alors qu'autrefois les travaux sur la "classe ouvrière" étaient beaucoup plus nombreux que ceux consacrés aux plus aisés, au cours des deux dernières décennies, la littérature sur la middle class s'est accrue de façon significative. Tout en étant manifeste dans les études explicitement consacrées aux classes sociales, comme le montrent les travaux de Richard H. Trainor, de Simon Gunn et de Robert J. Morris, cette tendance a plus largement affecté de nombreuses spécialités au sein de l'histoire sociale ${ }^{48}$.

Plusieurs facteurs peuvent expliquer la progression de l'intérêt historiographique pour les classes moyennes britanniques. Le charme accru de la bourgeoisie est en partie une réaction à la négligence de la période antérieure, dominée par les nombreux travaux sur l'aristocratie et sur la classe ouvrière. Mais ce changement a également été renforcé par les tournants linguistique et culturel. Les classes éduquées fournissent à l'historien une grande quantité de matériaux, que ce soient des romans, des journaux intimes, des lettres ou des peintures, qui se prêtent bien à la mise en ouvre d'approches discursives. L'intérêt croissant pour la consommation et la culture matérielle favorisait les études sur les classes possédantes. Les historiens du culturel semblaient souvent moins intéressés par les questions politiques (par exemple, sur l'histoire du mouvement ouvrier) qui avaient naguère été une motivation pour des étudiants issus des classes populaires. Bien sûr, il y a eu un travail important sur la culture populaire ces dernières années - le plus évident étant celui de Peter Bailey - et une conséquence importante de ce travail a été de remettre en cause cette idée que les classes et les cultures se recoupaient dans la GrandeBretagne du XIX ${ }^{\mathrm{e}}$ siècle ${ }^{49}$. Cependant, la prépondérance croissante des études sur la middle class au cours des deux dernières décennies est indéniable.

Les études sur la vie de la classe ouvrière dans la Grande-Bretagne du XIX ${ }^{\mathrm{e}}$ siècle n'ont pas disparu. Trevor Griffiths et Brad Beaven ont publié des analyses importantes au cours des dernières années. L'un et l'autre reflètent, quoique de façon différente, l'empreinte de tendances historiographiques récentes et la distance croissante à l'égard d'une lecture marxiste plus orthodoxe. Trevor Griffiths a prolongé la longue tradition de recherche sur la classe ouvrière du Nord-Ouest de l'Angleterre. Son travail complet, intitulé de façon signi-

48. Richard H. Trainor, "The middle class" dans Martin J. Daunton (ed.), The Cambridge Urban History of Britain, volume 3: 1840-1950, Cambridge, Cambridge University Press, 2000; Simon Gunn, The Public Culture of the Victorian Middle Class: Ritual and Authority in the English Industrial City, 1840-1914, Manchester, Manchester University Press, 2000; Robert J. Morris, Class, Sect and Party: the Making of the British Middle Class: Leeds, 1820-1850, Manchester, Manchester University Press, 1990.

49. Peter Bailey, Popular Culture and Performance in the Victorian City, Cambridge, Cambridge University Press, 1998. 
ficative au pluriel The Lancashire Working Classes («les classes laborieuses du Lancashire»), a cependant élargi la lecture «révisionniste» empirique du tableau dressé par Eric Hobsbawm, en révélant l'importance des distinctions de métier, de religion et d'origine ethnique, et en proposant un portrait plus individualiste de la vie des travailleurs ${ }^{50}$. Brad Beaven s'est lui tourné vers une région moins étudiée - Coventry - pour étudier les implications de l'âge, du genre et de la classe pour les jeunes travailleurs entre 1850 et $1945^{51}$. À certains égards, l'étude de Brad Beaven était "post-révisionniste» : elle prenait en compte les arguments révisionnistes, notamment ceux d'Andy Davies et de Joanna Bourke, sur les divisions entre travailleurs, mais elle cherchait à démontrer qu'une action plus collective était apparente quand les travailleurs étaient confrontés à des tentatives d'amélioration culturelle venues de la middle class, et insistait sur le caractère de classe de la culture plébéienne ${ }^{52}$. Par son intérêt pour les attitudes des jeunes travailleurs manuels spécialisés de sexe masculin, le livre de Brad Beaven intègre clairement l'influence du type de révisionnisme dans lequel s'inscrit le travail de Trevor Griffiths. Ce faisant, l'intérêt de Brad Beaven pour le loisir va dans le sens des travaux récents, bien que certaines de ses conclusions sur le caractère socialement exclusif de la culture populaire s'accordent mal avec les preuves qu'il fournit de la participation de gens rattachés, par leur activité, à la petite bourgeoisie. Si son travail a le mérite d'attirer l'attention sur la signification des rencontres culturelles entre les groupes sociaux, ses efforts pour démontrer que les cultures et les classes se recoupent étroitement dans la Grande-Bretagne du XIX ${ }^{\mathrm{e}}$ siècle ne sont pas toujours convaincants.

Le passage de l'histoire sociale à l'histoire culturelle est sans doute la tendance historiographique la plus discutée. La place réduite, et modifiée, des classes sociales dans les études sur le XIXe siècle illustre et encourage tout à la fois ce développement, qui était également manifeste dans la constitution et dans l'évolution de champs nouveaux (par exemple, l'histoire de la sexualité), ou dans des variantes plus traditionnelles de l'histoire sociale (comme l'histoire du sport). Ces travaux sont discutés par Rosalind Crone dans son article sur l'histoire culturelle dans ce numéro. Certains débats récents sur l'histoire sociale perçoivent l'explosion de l'histoire culturelle comme l'unique développement important dans la dernière génération, et pas seulement comme le principal. C'est cependant une image partielle, qui peut avoir des implications erronées pour les débats portant sur l'évolution de l'histoire britannique et son avenir. 2001 .

50. Trevor Griffiths, The Lancashire Working Classes c. 1880-1930, Oxford, Oxford University Press,

51. Brad Beaven, Leisure, Citizenship and Working-class Men in Britain, 1850-1945, Manchester, Manchester University Press, 2005.

52. Andrew Davies, Leisure, Gender and Poverty: Working-Class Culture in Salford and Manchester, 1900-39, Buckingham, Open University Press, 1992; Joanna Bourke, Working Class Cultures in Britain, 1890-1960: Gender, Class and Ethnicity, Londres, Routledge, 1994. 
On a soutenu que l'importance accordée à l'autonomie du politique - et même sur son pouvoir constitutif - avait eu des répercussions notables sur l'histoire sociale de la Grande-Bretagne du XIX ${ }^{e}$ siècle. Cependant, ce dont je souhaite traiter dans cette partie de mon article, c'est d'une relation souvent décrite comme celle d'une rupture consommée au cours des dernières années : le rapport entre l'histoire sociale et l'histoire économique. Au cours des années 1970, les relations étaient souvent étroites, et les travaux d'histoire sociale s'appuyaient couramment sur des quantifications. Au cours des années qui ont suivi, nombre de ces liens ont été rompus, et la quantification est devenue moins à la mode. Cependant, il reste des domaines importants de l'histoire sociale où la quantification reste essentielle. J'en examinerai ici deux : l'histoire démographique et l'histoire urbaine.

J'ai déjà noté que les approches interprétatives s'accommodaient rarement des aspirations à l'explication. L'histoire démographique du XIX ${ }^{e}$ siècle constitue un bon exemple d'un champ où les impératifs de l'explication ont gardé toute leur force. La transition d'un régime à haute pression démographique (forte natalité, forte mortalité) à un régime de basse pression (faible natalité, faible mortalité) garantissait que les historiens de la population du XIX ${ }^{e}$ siècle se consacrent à l'explication de ce changement de régime. Les deux aspects de cette transition ont inspiré des travaux importants ces dernières années, dont celui de Simon Szreter. Dans son étude monumentale Fertility, Class and Gender in Britain, 1860-1940, Simon Szreter s'est beaucoup appuyé sur des données quantitatives pour essayer de modifier les interprétations établies de la relation entre la classe sociale et la taille de la famille en Grande-Bretagne ${ }^{53}$. Il l'a en partie fait en démontrant que les travaux existants surestimaient la corrélation entre le statut social élevé et la réduction de taille de la famille. Ses nouveaux calculs reposaient cependant sur une démarche d'histoire intellectuelle. Il démontrait que les catégories de la profession et de la classe du recensement de 1911 - sur lequel reposaient l'hypothèse diffusionniste (le «modèle professionnel») selon laquelle la réduction de la taille de la famille descendait des classes sociales élevées vers les classes populaires - étaient elles-mêmes forgées par des postulats sur la relation entre mérite social et petite taille de la famille. Ces postulats faisaient beaucoup pour inscrire les conclusions dans les données elles-mêmes.

Plus récemment, Simon Szreter s'est intéressé à l'autre volet de la transition démographique : la baisse des taux de mortalité ${ }^{54}$. Il a contesté l'idée que la croissance économique elle-même crée une santé publique meilleure.

53. Simon R. S. Szreter, Fertility, Class and Gender in Britain, 1860-1940, Cambridge, Cambridge University Press, 1996.

54. Simon R. S. Szreter, Health and Wealth: Studies in History and Policy, Rochester (New York), Boydell \& Brewer, 2005. 
Revisitant la mortalité dans les cités britanniques de la première moitié du XIX ${ }^{\mathrm{e}}$ siècle, Simon Szreter soutient que les effets de l'industrialisation ont bien failli être catastrophiques, ramenant les espérances de vie dans certains quartiers au niveau du XIVe siècle. L'industrialisation a entraîné une perturbation, qui a entrâné des privations, des maladies et des décès. Ce n'est qu'après 1870, quand les hommes politiques locaux ont commencé à forger des politiques plus interventionnistes, que les ravages du changement économique ont été limités. Très attaché à la quantification, et profondément préoccupé par l'économie, Simon Szreter dans son histoire démographique démontre la fécondité maintenue et la variété de l'histoire sociale en GrandeBretagne.

Simon Szreter a été un des contributeurs au volume de la Cambridge Urban History of Britain consacré au XIX ${ }^{e}$ siècle et dirigé par Martin Daunton. Comme le notait Peter Clark, le responsable de l'ensemble de l'ouvrage, nombre des pionniers en histoire urbaine sont des spécialistes de l'époque victorienne ${ }^{55}$. Premier professeur d'histoire urbaine de Grande-Bretagne, Jim Dyos a joué un rôle majeur dans le développement de ce champ. Les premiers travaux de Jim Dyos portaient sur la banlieue londonienne de Camberwell à l'époque victorienne. Promoteur important de la culture de travail en équipe, Dyos co-dirigea un ouvrage-clé dans l'histoire sociale de la ville victorienne ${ }^{56}$. C'est cependant l'ouvrage d'Asa Briggs, Victorian Cities, paru pour la première fois en 1963, qui fut peut-être le livre majeur qui a nourri l'essor de l'histoire des villes au XIX ${ }^{\mathrm{e}}$ siècle. Organisé autour d'une série d'études de cas incluant des chapitres fameux sur Manchester, Middlesborough et Melbourne, le livre célèbre d'Asa Briggs explore ingénieusement la dynamique et l'impact de l'urbanisation sur la Grande-Bretagne du $\mathrm{XIX}^{\mathrm{e}}$ siècle et au-delà ${ }^{57}$. L'exemple de Jim Dyos et d'Asa Briggs fut suivi par un certain nombre d'héritiers éminents, dont Philip Waller, David Cannadine et Martin Daunton ${ }^{58}$. Les années 1970 connurent ainsi un développement de l'histoire urbaine, qui s'intéressait résolument à la fois à l'économique et au spatial, mais qui aspirait, dans ce qu'elle avait de plus optimiste, à offrir une perspective panoramique de la vie d'une ville. Dans sa longue introduction au volume de la Cambridge Urban History qu'il a dirigé, Martin Daunton affirme que ces ambitions étaient en perte de vitesse. En 2000,

55. Peter Clark in Martin Daunton (ed.), The Cambridge Urban History, ouv. cité, p. xxi.

56. H. J. Dyos, Victorian Suburb: a Study of the Growth of Camberwell, Leicester, Leicester University Press, 1961 ; H.J. Dyos and Michael Wolff (eds.), The Victorian City: Images and Realities, Londres, Routledge 1973, 2 volumes.

57. Asa Briggs, Victorian Cities, Londres, Odhams, 1963.

58. Philip. J. Waller, Democracy and Sectarianism: a Political and Social history of Liverpool, 18681939, Liverpool, Liverpool University Press, 1981; Philip J. Waller, Town, City and Nation: England 1850-1914, Oxford, Oxford University Press, 1983; David Cannadine, Lords and Landlords: the Aristocracy and the Towns, 1774-1967, Leicester, Leicester University Press, 1980; Martin J. Daunton, Coal metropolis: Cardiff 1870-1914, Leicester, Leicester University Press, 1977. 
Martin Daunton détectait à juste titre le retour à un ton plus assuré. Cette renaissance urbaine est à noter.

Comme l'indiquait le sommaire du volume dirigé par Martin Daunton (plus que la Cambridge Social History plus ancienne de F.M.L. Thompson), l'histoire urbaine a assurément pris toute la mesure du tournant culturel. A partir des années 1990, des études importantes sur la culture urbaine, comme celles de Judith R. Walkowitz et Lynda Nead, ont vu le jour ${ }^{59}$. L'histoire urbaine a cependant préservé un rapport ferme tant avec l'économique qu'avec le social, et un intérêt prononcé pour le fonctionnement tant du pouvoir local que du gouvernement central. Comme pour la démographie, cela reflétait la place centrale occupée par les processus dans son identité de sousdiscipline. La compréhension de la périodisation, du caractère et des effets de l'urbanisation a fourni à l'histoire urbaine du XIX $\mathrm{X}^{\mathrm{e}}$ siècle sa définition et son ambition. Cela pouvait, bien sûr, donner naissance à différentes formes de téléologie, à une fausse logique de processus, ou à des notions réifiées de "modernité» et de "modernisation», mais cela avait la vertu d'installer la diachronie et la comparaison au cœur du projet.

La propre contribution de Martin Daunton à la Cambridge Urban History transmettait une certaine vitalité de l'histoire urbaine, ainsi que les possibilités qui demeuraient pour des emprunts féconds à l'économie et à la sociologie. L'économie politique figurait en bonne place dans l'analyse faite par Martin Daunton des groupes en concurrence qui s'occupaient des services municipaux, en particulier ceux qui reposaient sur des monopoles naturels. En s'intéressant à la structure de gestion relativement faible d'une bonne part de l'économie urbaine britannique, Martin Daunton, influencé par Robert J. Morris, mettait l'accent sur le rôle des facteurs externes montré par l'analyse des réseaux et par le recours au capital social ${ }^{60}$. Cette ouverture d'esprit théorique, caractéristique du travail de Martin Daunton, a été plus féconde dans certains domaines que dans d'autres. Le concept de capital social utilisé par Simon Szreter et par Martin Daunton peut poser problème. Même en utilisant une subtile distinction supplémentaire entre le capital qui comble un fossé et celui qui exclut, la notion de capital demeure un instrument trop brut pour saisir la complexité et les conflits des relations sociales. Par exemple, il est clair que certaines collectivités (les Églises par exemple) peuvent représenter à la fois un capital qui relie (si on les considère en termes de classes sociales) et qui exclut (si on les considère en termes confessionnels). Il ne faut pas surestimer le potentiel d'explication du capital social, ni son

59. Judith R. Walkowitz, City of Dreadful Delight: Narratives of Sexual Danger in Late Victorian London, Chicago, University of Chicago Press, 1992; Lynda Nead, Victorian Babylon: Peoples, Streets and Images in Nineteenth-Century London, New Haven, Yale University Press, 2000; Deborah Epstein Nord, Walking the Victorian Streets: Women, Representation and the City, Ithaca (N.Y.), Cornell University Press, 1995.

60. Robert J. Morris, "Externalities, the market, power structure and the urban agenda", Urban History Yearbook, $\mathrm{n}^{\circ} 17,1990$, p. 99-109. 
indépendance à l'égard des catégories sociales plus anciennes. Néanmoins, pris dans son ensemble, le volume de Martin Daunton ouvre avec force pour la vitalité de l'histoire urbaine et pour le maintien des liens entre l'histoire sociale et l'histoire économique.

On peut voir de multiples façons l'évolution de l'histoire sociale britannique au cours des trente dernières années. On l'a peinte comme le cas paradigmatique de l'ascension et de la chute d'une sous-discipline, ou bien comme un exemple réussi de revigorante réinvention. Elle a certainement connu une prolifération accrue de ses sous-champs, rendant quasi impossible un travail synoptique dans un format concis. Cet article a cherché à prendre en compte l'étendue des remises en cause méthodologiques, leurs tensions parfois, et d'en restituer certaines conséquences. Il a, cependant, également cherché à se départir d'une certaine façon du récit dominant sur l'ascension de l'histoire culturelle, en revenant sur les relations entre l'histoire «sociale», l'histoire "politique» et l'histoire "économique» au cours des trente dernières années.

Dans son étude récente des mouvements historiographiques au cours des quarante dernières années, Geoff Eley demande aux historiens de garder, sinon les ambitions totalisantes de la nouvelle histoire sociale des années 1960, du moins son aspiration à poser de grandes questions, et à établir des liens ${ }^{61}$. En cela, il a sûrement raison. La plus grande sophistication théorique que nous héritons des trente dernières années ne doit empêcher ni l'ambition ni l'explication. Les preuves existent qu'on cherche davantage à combiner les idées et les démarches de l'histoire sociale et de l'histoire culturelle, mais il faudrait sans doute en élargir les horizons pour obtenir des progrès véritables ${ }^{62}$. Il nous faut éviter les modèles monolithiques et auto-réalisants de la "modernisation", que ceux-ci prennent une forme libérale optimiste ou foucaldienne pessimiste. Nous devons aussi reconnaître que le langage contemporain de l'interdisciplinarité n'est pas sans créer ses propres exclusions. Cette confrontation plus large est essentielle si nous voulons ranimer la curiosité autocritique et l'ouverture intellectuelle du meilleur de la nouvelle histoire sociale.

James Thompson est reader en histoire contemporaine à l'université de Bristol

Traduit de l'anglais par Fabrice Bensimon, avec le concours de Robert Tombs

61. Geoff Eley, A Crooked Line, ouv. cité, p. 203.

62. Ibidem; William H. Sewell, Logics of History, ouv. cité; Geoff Eley and Keith Nield, The Future of Class in History: What's Left of the Social?, Ann Arbor (Michigan), University of Michigan Press, 2007. 